\title{
A Liquid-Phase Quartz Crystal Microbalance for Photovoltaics Research
}

Craig L. Perkins and Xiaonan Li

National Renewable Energy Laboratory, Golden, CO 80401

NREL/PR-520-43324

Presented at the 33rd IEEE Photovoltaic Specialist Conference held May 11-16, 2008 in San Diego, California 

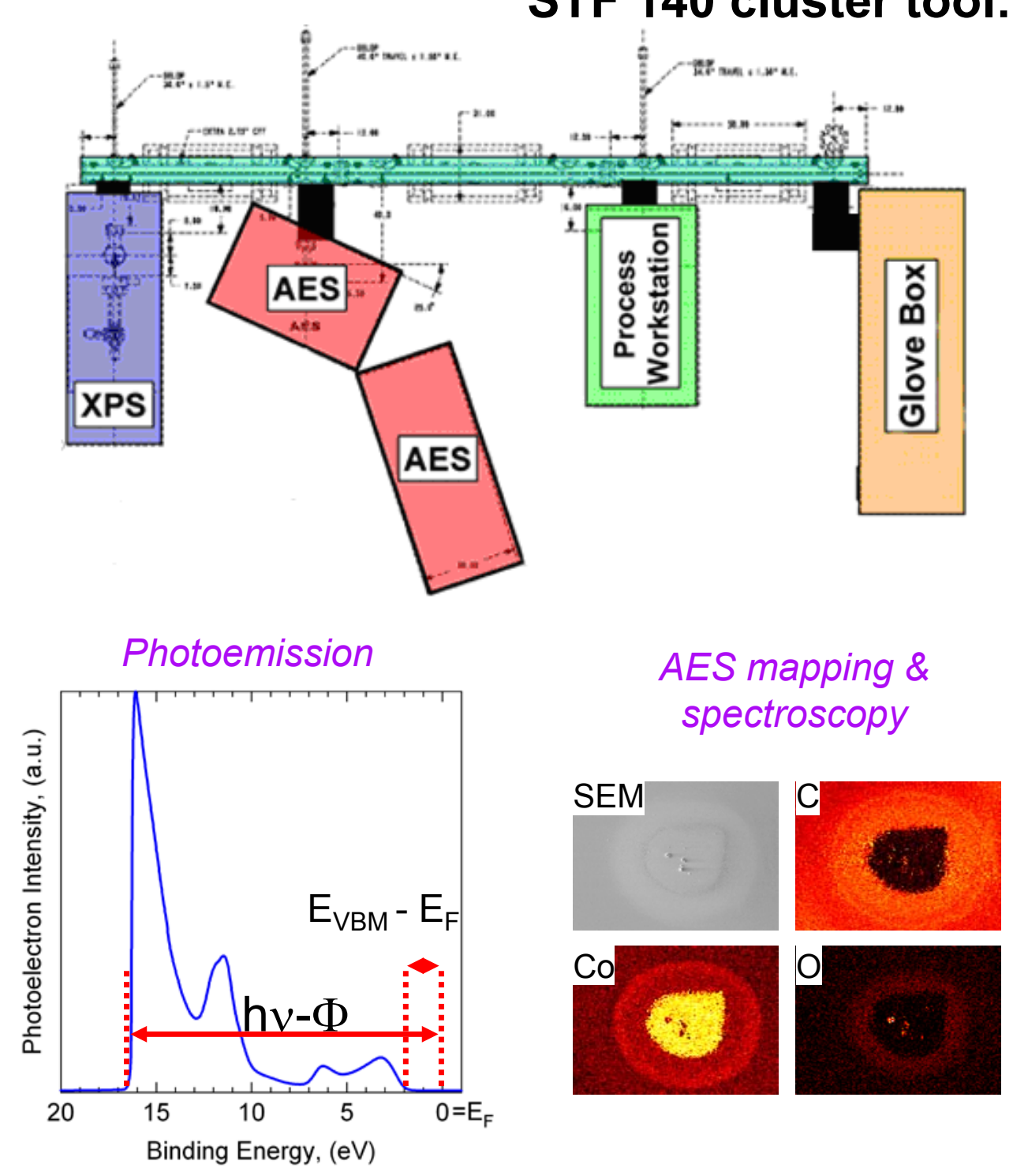

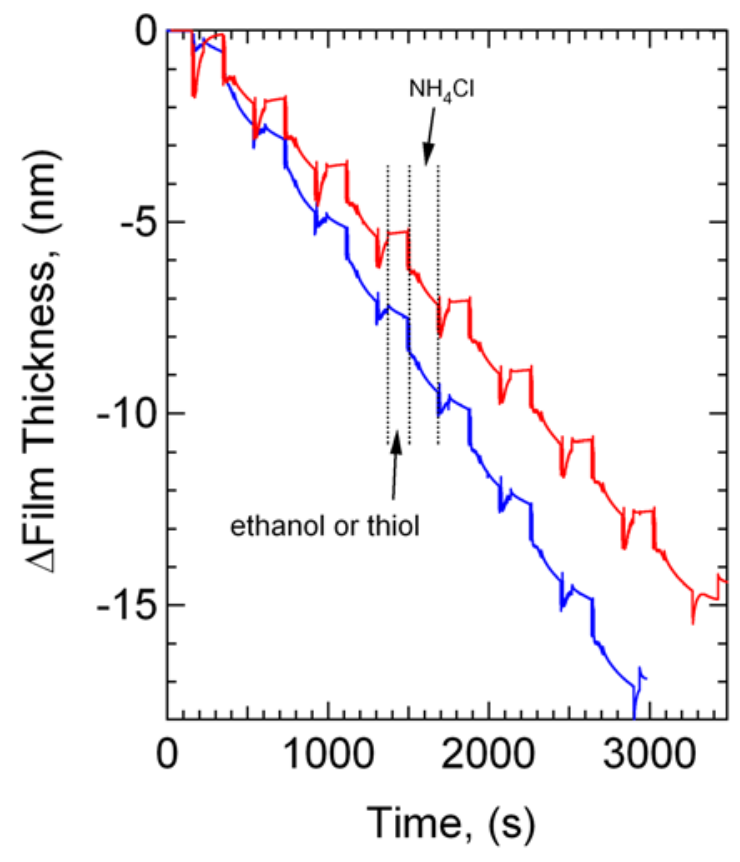

controlled ambient transfer, QCM, chemical growth, etching, \& modification

M. Law et al. "The Structural, Optical and Electrical Properties of PbSe Nanocrystal Solids Treated Thermally and with Simple Amines", J. Am. Chem. Soc.,(2008) ASAP article.

J.M. Luther et al.,"The Structural, Optical, and Electrical Properties of SelfAssembled Films of PbSe Nanocrystals Treated with 1,2-ethanedithiol", ACS Nano, 2 (2008) 271-280.

G. Teeter, "X-ray and ultraviolet photoelectron spectroscopy measurements of Cu-doped CdTe(111)-B: Observation of temperature-reversible CuxTe precipitation and effect on ionization potential", J. Appl. Phys., 102 (2007) 034504. 


$$
\Delta f=-f^{3 / 2} \sqrt{\frac{\eta_{L} \cdot \rho_{L}}{\pi \cdot \mu_{q} \cdot \rho_{q}}}
$$

\section{QCM-Based Flow Reactor Schematic}

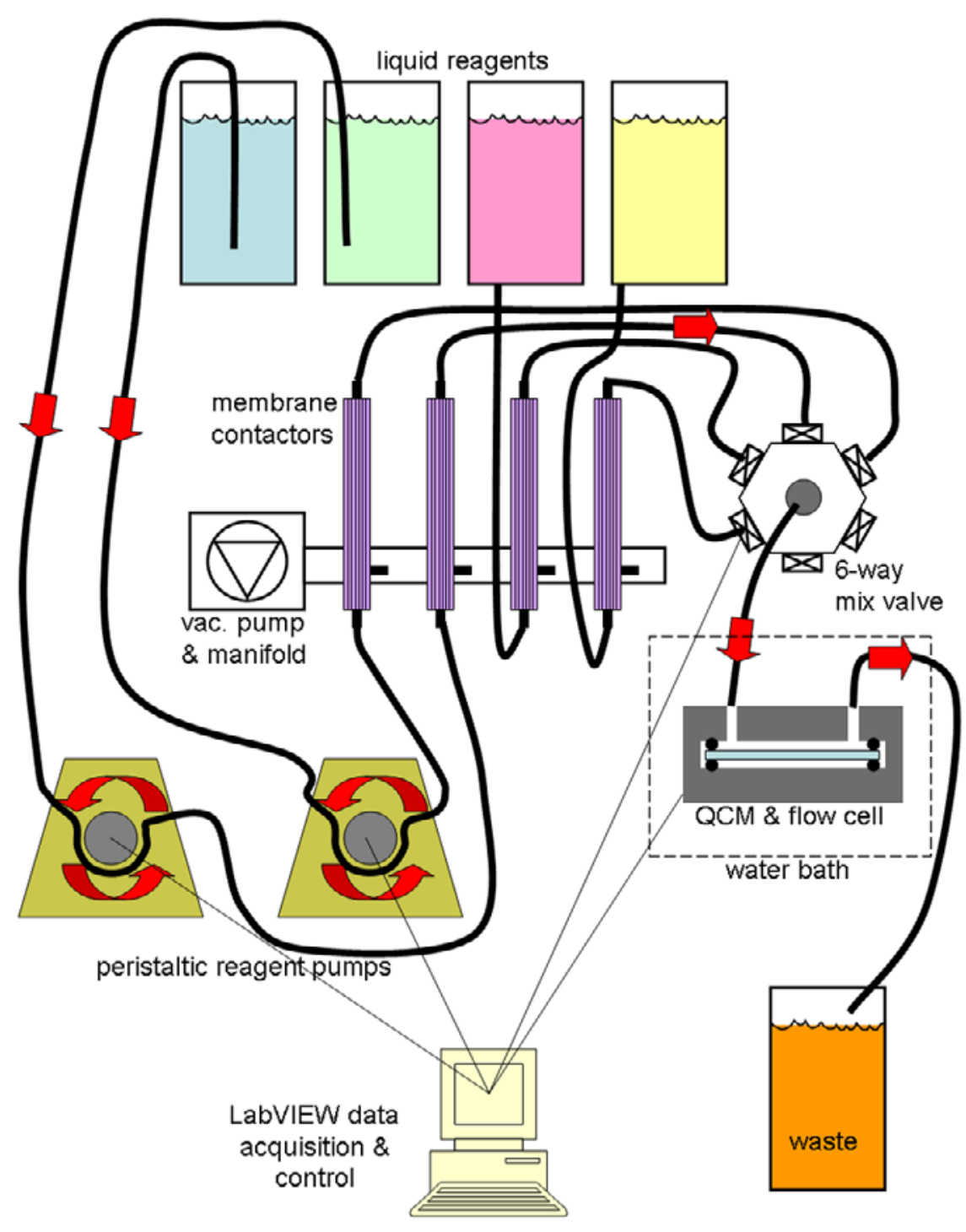

K. K. Kanazawa and J. G. Gordon, Analytica Chimica Acta 175, 99 (1985). 
$\mathrm{NH}_{4} \mathrm{Cl}$ : Using an Etchant as a Probe of ZnO-molecule Interactions
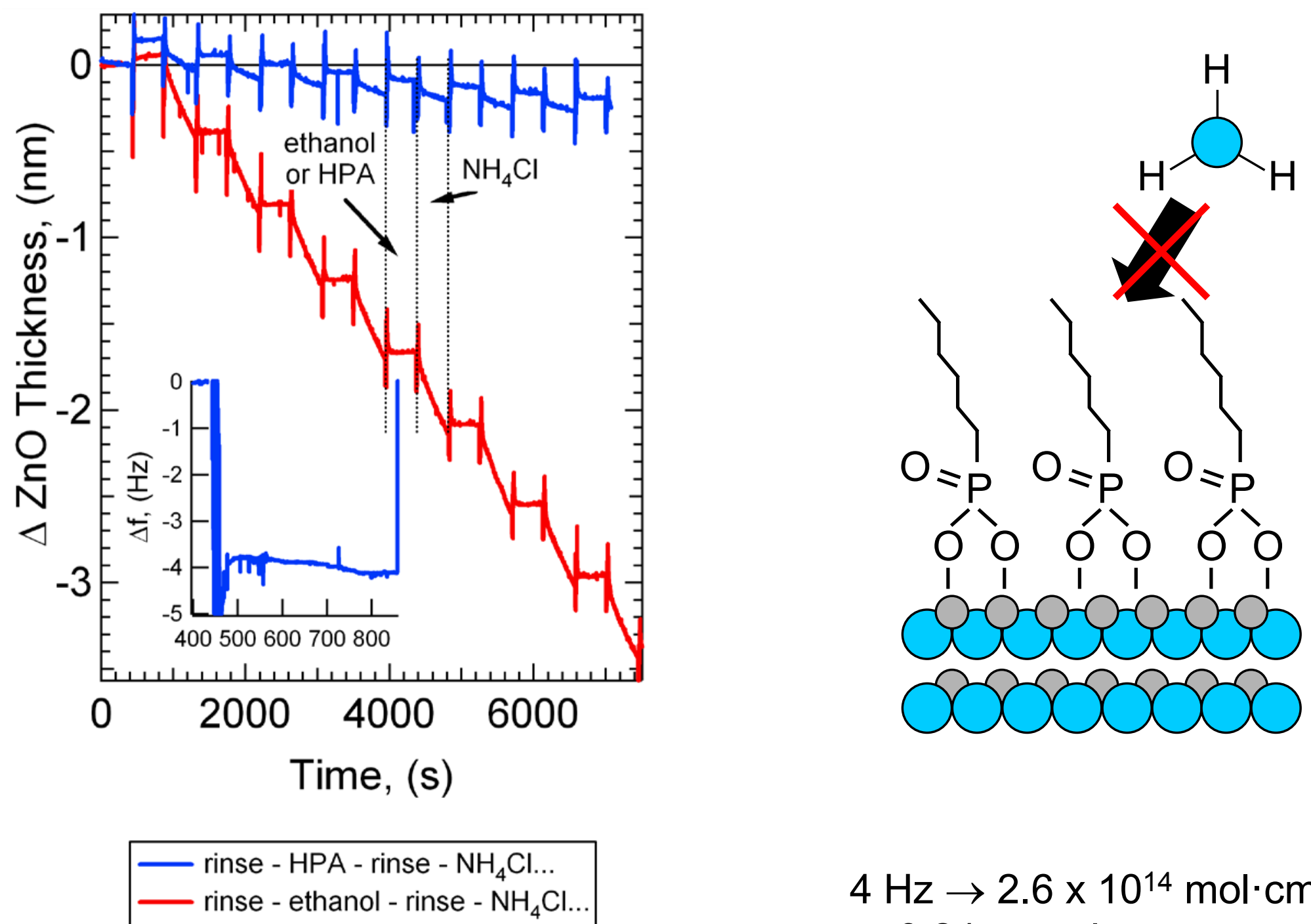

$4 \mathrm{~Hz} \rightarrow 2.6 \times 10^{14} \mathrm{~mol} \cdot \mathrm{cm}^{-2}$, or 0.24 monolayer. 


\section{QCM Thickness Data for CdS Chemical Depositions}
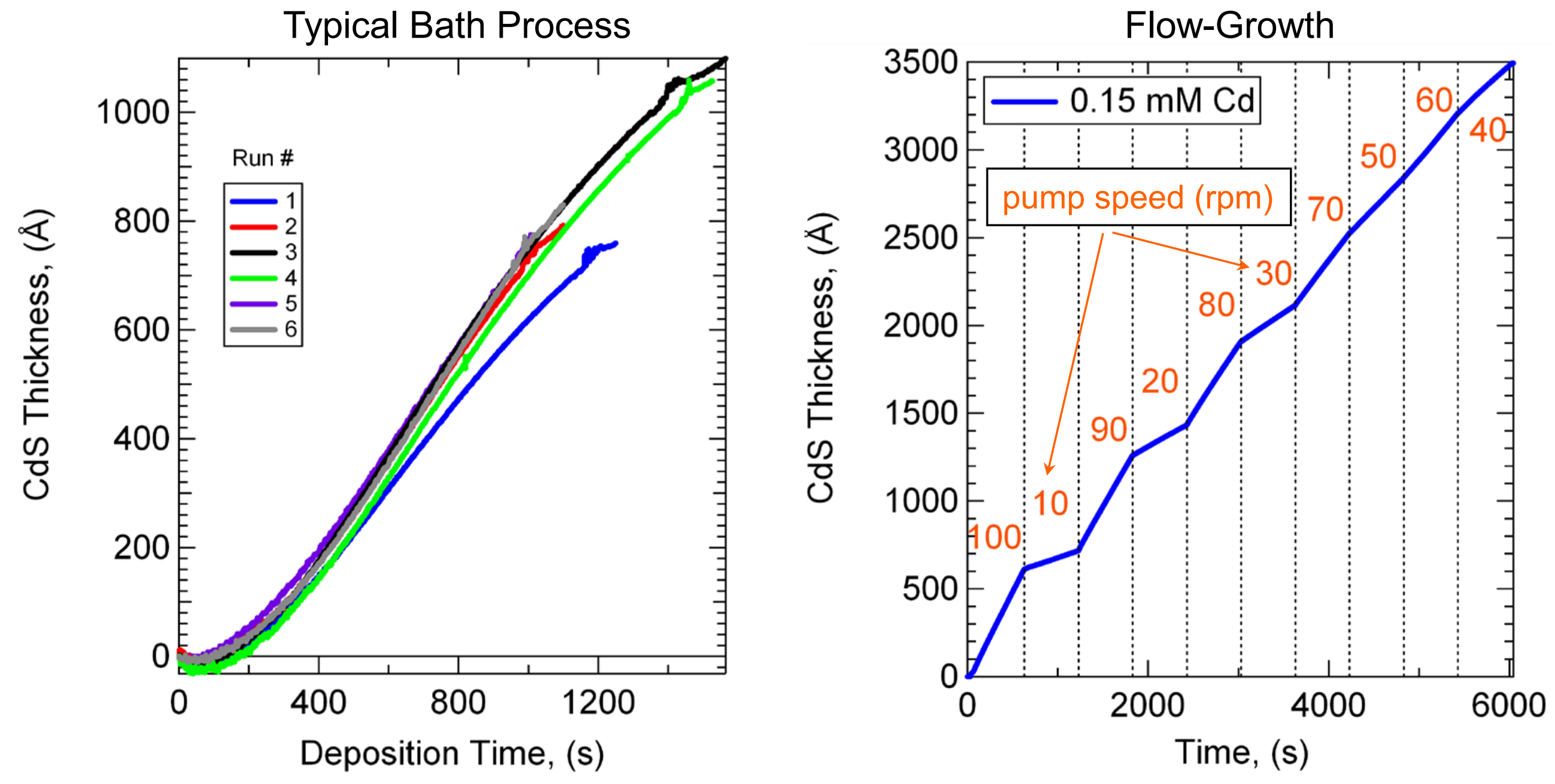
Deposition Rates as f(flow, [reactants])

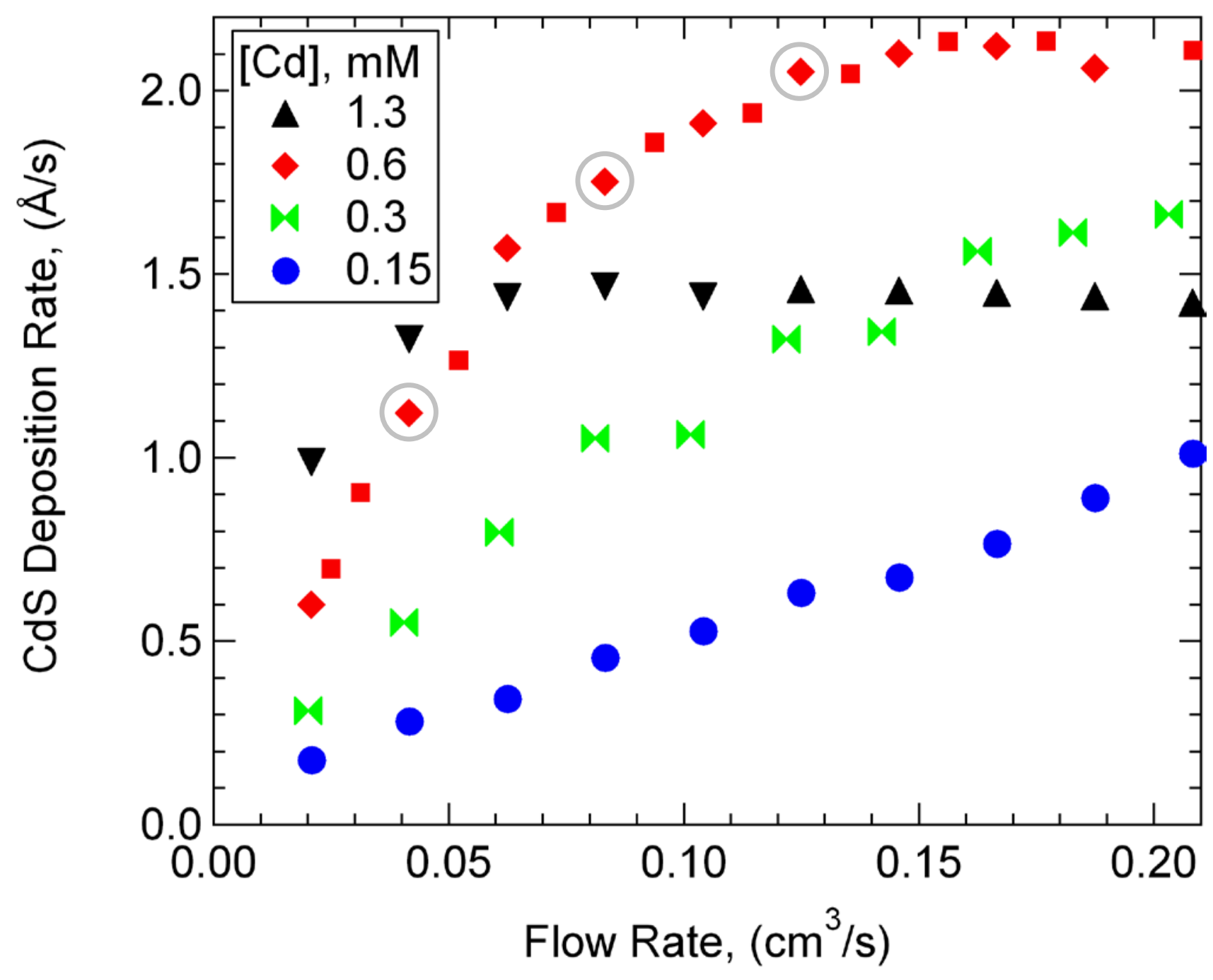




\section{Flow-Rate Dependent Morphology of Chemically Grown CdS Films}

Film thickness $=0.4 \mu \mathrm{m}$

$\mathrm{T}_{\text {growth }}=63^{\circ} \mathrm{C}$
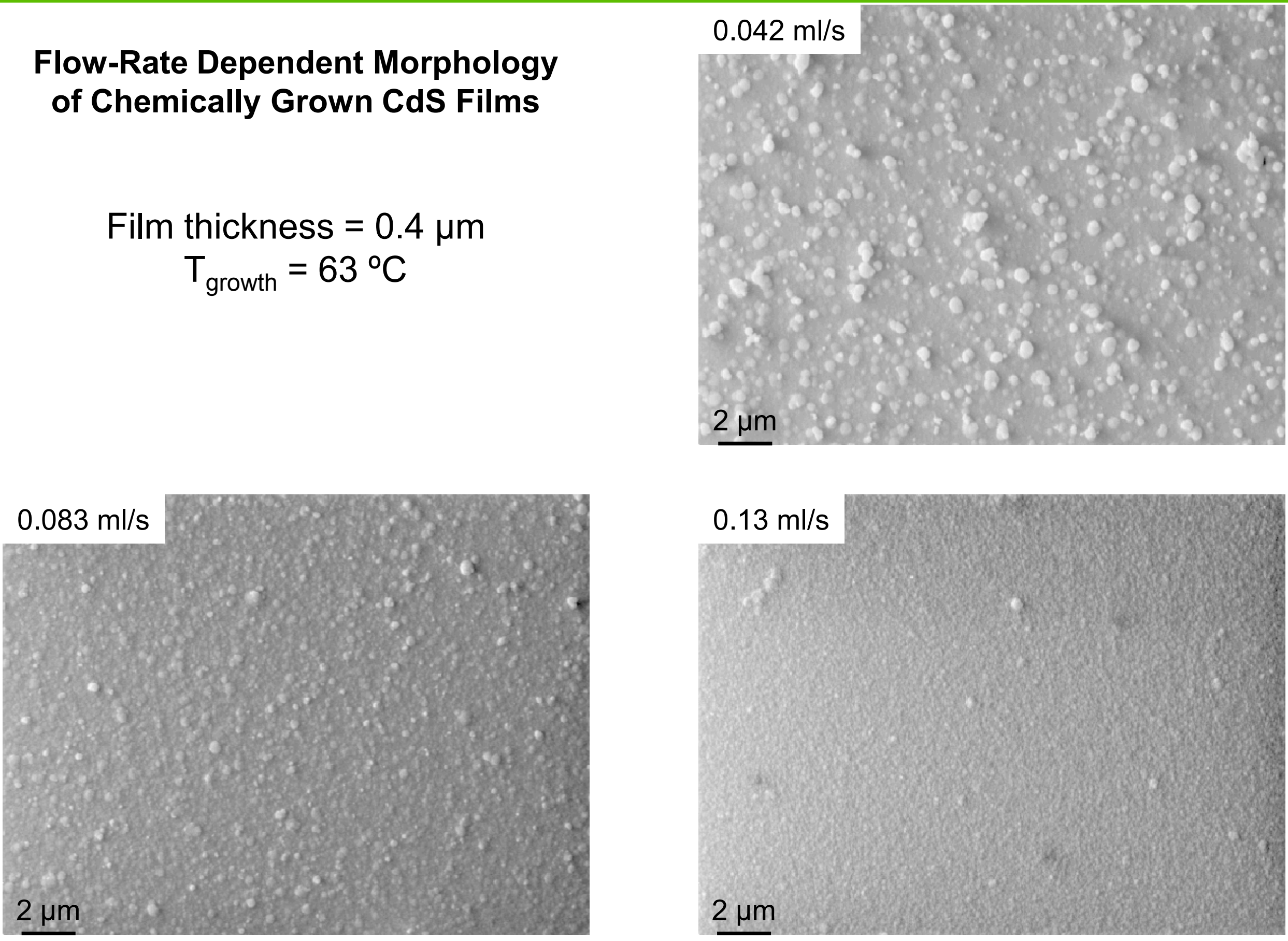


\section{Summary}

- Construction of a new QCM-based flow reactor has been completed - Initial results include:

- Stable oscillation achieved with crystals sputter coated at $200^{\circ} \mathrm{C}$ with $500 \mathrm{~nm} \mathrm{ZnO}$

- Sub-Hz noise level means < < monolayer sensitivity

- Operation at elevated temperature possible after installation of membrane contactors

- Determination of $\mathrm{ZnO}$ etch rates using dilute ethanolic $\mathrm{NH}_{4} \mathrm{Cl}$

- Demonstration of use of an etchant as a probe of oxide-molecule interactions.

- Use in flow rate-dependent CdS chemical deposition

\section{Acknowledgments:}

Xiaonan Li, Sally Asher, Glenn Teeter, Pete Sheldon 
Contact Information:

Craig L. Perkins

National Renewable Energy Laboratory

1617 Cole Blvd., MS 3215

Golden, CO 80401 USA

(303) 384-6659 [office]

(303) 384-6564 [lab]

(303) 384-6604 [fax]

craig_perkins@nrel.gov 